\title{
RE-ENTRAINMENT OF SMALL AGGREGATE PARTICLES FROM A PLANE SURFACE BY AIR STREAM
}

\author{
YASUO KOUSAKA, KIKUO OKUYAMA \\ AND YOSHIYUKI ENDO \\ Department of Chemical Engineering, University of \\ Osaka Prefecture, Sakai 591
}

\begin{abstract}
A basic study of re-entrainment of dust particles from collecting media of dust collectors by air stream has been made. It is pointed out in the analysis of the re-entrainment mechanism that the bending stress induced by drag plays an important role and that the critical diameter of particle to be re-entrained is proportional to $-2 / 3$ power of velocity gradient. It is suggested from experiment that aggregate particles, once collected on the collecting media, are fairly stable in usual operation of dust collectors where velocity gradient is not so large.
\end{abstract}

\section{Introduction}

Dust particles deposited on collecting media of dust collectors, such as fibers, droplets, plates and so forth, should not be re-entrained by the air stream around the media. Basic studies of re-entrainment of fine dust particles from collecting media are so far very few, in spite of the importance of phenomena which decrease the efficiency of a dust collector, though many sudies have been made of re-entrainment of large particles from the bottom of a river, from road surfaces or from floors by fluid flow ${ }^{2,7}$. The essential difference between re-entrainment of large particles and of small ones exists in the mechanism of resistance against fluid drag, since the weight of a particle itself plays an important role in the former case while the adhesive force plays an important role in the latter.

The analysis and the experimental work in this paper is aimed at understanding whether or not re-entrainment has a significant effect on dust collection.

\section{Basic Consideration}

Re-entrainment of an aggregate particle from a plane surface by an air stream will occur when external force acting on the particle exceeds the adhesive force of the particle to the plane surface or exceeds the strength of the aggregate particle. In this section, the mechanism of re-entrainment of an aggregate particle deposited on a flat glass wall and exposed to an air stream is discussed.

\section{1 External force acting on a particle}

It is assumed in the following analysis that the par-

Received May 4, 1979. Correspondence concerning this article should be addressed to $\mathrm{Y}$. Kousaka. ticle is spherical and is small enough to be held inside the laminar sub-layer in which the velocity gradient of air flow is almost linear. The drag force, or external force, acting on a small segment, $f(y) d y$ shown in Fig. 1, is approximated here as follows, when Stokes' law is applied to the flow around the particle.

$$
d R_{f}=\frac{24 \mu}{u d_{p} \rho_{f}} f(y) d y \frac{\rho_{f} u^{2}}{2}=\frac{24 \mu \gamma}{d_{p}} \sqrt{\left(d_{p}-y\right) y} y d y
$$

The integration of Eq. (1) under $u=$ const gives the Stokes drag, $3 \pi \mu u d_{p}$.

The bending stress induced by the drag force at the distance $y^{*}(>0)$ above the flat surface, shown in Fig. 2 (a), is given as (see Appendix):

$$
\begin{aligned}
\sigma_{b} & =\frac{96 \mu \gamma}{\pi} \int_{y^{*} / d_{p}}^{1} \sqrt{(1-Y) Y} Y^{2} d Y\left[\frac{y^{*}}{d_{p}}\left(1-\frac{y^{*}}{d_{p}}\right)\right]^{-3 / 2} \\
& \simeq \frac{3.75 \mu}{y^{* 3 / 2}} \gamma d_{p}^{3 / 2} \propto \gamma d_{p}^{3 / 2} \quad\left(y^{*} \ll d_{p}\right)
\end{aligned}
$$

And the shearing stress is given as (see Appendix):

$$
\begin{aligned}
& \tau=\frac{24 \mu \gamma}{\pi} \int_{y^{*} / d_{y}}^{1} \sqrt{(1-Y)} \bar{Y} Y d Y\left[\frac{y^{*}}{d_{p}}\left(1-\frac{y^{*}}{d_{p}}\right)\right]^{-1} \\
& \simeq \frac{1.5 \mu}{y^{*}} \gamma d_{p} \propto \gamma d_{p} \quad\left(y^{*} \ll d_{p}\right)
\end{aligned}
$$

In comparison of the above equations, it can be found that the bending stress is much higher than the shearing stress. The bending stress, as is well known, is composed of tensile and compressive stresses. The compressive stress, however, may not contribute to separating or re-entraining an aggregate. As a result, when the bending stress is increased the neutral plane of the bending stress will actually move toward the compres- 


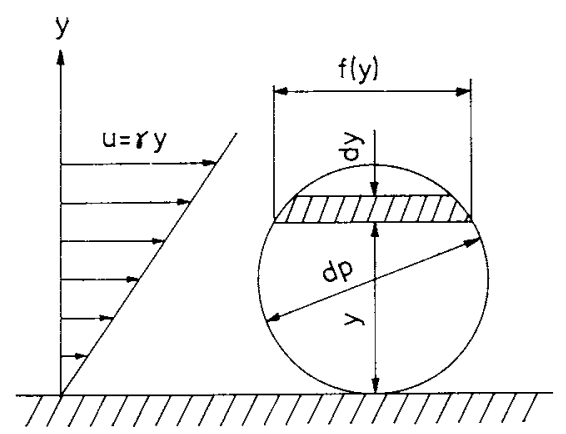

Fig. 1 Velocity profile and a particle

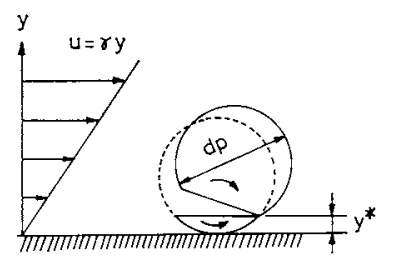

(a)

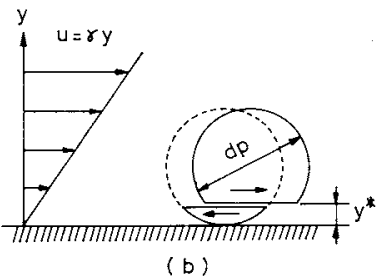

Fig. 2 Re-entrainment by bending and shearing stress

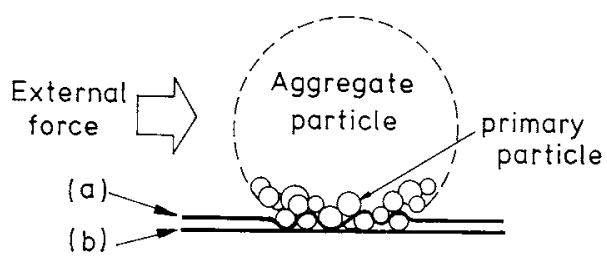

Fig. 3 Position where an aggregate particle is separated

sive side, and the break-up or re-entrainment of the aggregate will be caused mainly by the tensile stress.

The tensile strength of an aggregate, on the other hand, is expected to be of the same order as the shearing strength or less, since these strengths are the resultant of cohesive and frictional forces between particles. When the cohesive force is larger than the frictional force, as it may be in most cases for fine particles, the tensile and shearing strengths are expected to be almost the same because of the same mechanism of the break-up.

Thus it is concluded that re-entrainment of aggregates in laminar sub-layer is caused principally by bending force.

The value of $y^{*}$ appears in the above equations is difficult to evaluate, but it is expected to be very close to the flat surface. Since both the bending moment and the shearing force increase with a decrease of distance $y$ shown in Fig. 1, while the sectional area of the particle decreases with a decrease of $y$, the bending and shearing stresses increase sharply with a decrease of $y$ in the vicinity of the flat surface. The value of $y^{*}$ is defined as the mean distance where the above stresses just exceed the strength of an aggregate.

\subsection{Strength of an aggregate particle}

It is difficult to evaluate the strength of an aggregate particle, and there is no available equation for such evaluation except Rumpf's relation ${ }^{6}$, which was derived for the strength of a powder bed resulting from water bridge, shown as follows:

$$
\sigma_{p}=\frac{(1-\varepsilon) k}{\pi d_{p 1}} \sigma F_{H}
$$

The adhesive force of an aggregate particle to a flat surface, on the other hand, is also important if the particle is re-entrained just at the surface $\left(y^{*}=0\right)$. This force, however, is essentially induced in the same way as that in an aggregate particle regarding the force resulting from water bridge. It is reported that the adhesive force of a spherical particle to a flat surface is twice that induced between two similar spherical particles $^{13}$. Considering the existence of electrical force, the adhesive strength of an aggregate particle to a flat surface is expected to be greater than that of the aggregate particle.

Figure 3 illustrates the manner of two possible separations according to the above two cases. This will be discussed in a later section.

\section{3 Critical particle diameter to be re-entrained}

If the separation occurs in an aggregate particle shown as (a) in Fig. 3, which will be discussed later, and if the strength of the particle can be evaluated by Eq. (6), the critical particle diameter to be re-entrained can be given by combining Eq. (6) with Eqs. (2) and (3) as

$$
d_{50} \propto \gamma^{-2 / 3}
$$

\section{Experimental}

\section{1 Apparatus and method}

The powders used in the experiments are shown in Table 1. Aggregate particles were generated by a rotating blade-type generator ${ }^{4}$, and they were sampled on a glass plate by means of gravitational settling. Then the glass plate, on which aggregates are deposited, is installed in the observation cell upsidedown as shown in Fig. 4. The cross-section of the cell is $5.5 \mathrm{~mm}$ in width and $3 \mathrm{~mm}$ in depth, and the bottom wall is composed of a glass plate so that the particles are illuminated through it from the bottom. Thusilluminated particles were observed on a TV monitor through a microscope, as shown in Fig. 4.

Clean air was introduced into the cell and the flow 
Table 1 Properties of aggregate particles

\begin{tabular}{|c|c|c|c|c|c|c|c|c|}
\hline Key & Calculated & Particles & {$\left[\begin{array}{c}\mathrm{ep} \\
{\left[\mathrm{g} / \mathrm{cm}^{3}\right]}\end{array}\right]$} & {$\left[\begin{array}{l}d p: \\
{[\mu]}\end{array}\right.$} & {$[-]$} & {$\left[\begin{array}{c}k \\
{[-]}\end{array}\right.$} & $\begin{array}{c}M f \\
{[w t \%]}\end{array}$ & {$\left[10^{5} \sigma_{p}^{\sigma_{p}} / \mathrm{cm}^{2}\right]$} \\
\hline $\mathrm{O}$ & & \multirow{2}{*}{ Bentonite } & \multirow{2}{*}{2.6} & \multirow{2}{*}{0.51} & \multirow{2}{*}{0.71} & \multirow{2}{*}{3.47} & 5.82 & 9.05 \\
\hline 0 & - & & & & & & 13.12 & 8.14 \\
\hline - & & \multirow{2}{*}{ Calcium Carbonate } & \multirow{2}{*}{2.6} & \multirow{2}{*}{0.64} & \multirow{2}{*}{0.66} & \multirow{2}{*}{4.04} & 0.12 & 13.3 \\
\hline- & $\cdots$ & & & & & & 7.17 & 9.84 \\
\hline$\Phi$ & & \multirow{2}{*}{ Clay } & \multirow{2}{*}{2.6} & \multirow{2}{*}{0.76} & \multirow{2}{*}{0.86} & \multirow{2}{*}{1.91} & 0.24 & 1.98 \\
\hline$-\infty$ & $-\cdots$ & & & & & & 14.58 & 1.37 \\
\hline 0 & - & Acid Activated Clay & 2.6 & 0.99 & 0.81 & 2.41 & 12.97 & 1.86 \\
\hline$\square$ & & \multirow{2}{*}{ Jitanium Dioxide } & \multirow{2}{*}{4.5} & \multirow{2}{*}{0.124} & \multirow{2}{*}{0.88} & \multirow{2}{*}{1.72} & 0.39 & 8.97 \\
\hline$-\square$ & & & & & & & 2.49 & 7.25 \\
\hline$\Delta$ & -...- & Iron Oxide $\left(\gamma-\mathrm{Fe}_{2} \mathrm{O}_{3}\right)$ & 5.2 & 0.08 & 0.90 & 1.53 & 1.06 & 8.99 \\
\hline$\Delta$ & $--\longrightarrow$ & Iron Oxide $\left(\alpha-\mathrm{Fe}_{2} \mathrm{O}_{3}\right)$ & 5.2 & 0.31 & 0.86 & 1.91 & 0.20 & 4.75 \\
\hline & & \multicolumn{7}{|c|}{ Thickness of laminar sub-layer } \\
\hline
\end{tabular}

arrived at the observing section after a sufficient distance to develop the flow, about $20 \mathrm{~cm}$. The velocity gradient at the observing section was evaluated by the von Karman equation ${ }^{3}$ ) for the velocity distribution of turbulent flow and the Blasius equation for the friction factor, which is given as

$$
\gamma=\frac{0.0791\left(D_{e} / \nu\right)^{-1 / 4}}{2 \nu} \bar{u}^{7 / 4}
$$

where $D_{e}$ is an equivalent diameter of the cell. The size distribution of aggregates was measured in Feret diameter. The air humidity ranged between $70 \%$ and $80 \%$. In this range no effect of humidity change on the results was found.

\subsection{Experimental results and discussion}

It was observed that large particles were first reentrained with increase of air flow rate and then smaller particles came to be re-entrained with successive increase of flow rate. This is reasonable as is indicated by Eq. (7). It was also observed in most experiments that re-entrainment took place in the way shown in Fig. 5, that is, small particles were left on the glass wall after re-entrainment of large particles. This suggests that the separation occurs not at the glass surface but inside the aggregate, shown as (a) in Fig. 3. The distance from flat surface, $y^{*}$, at which separation takes place, is also suggested to be very small.

Figure 6 is an example of the experimental results. It is seen in the figure that the mode diameter decreases with an increase of mean air velocity in the cell and that total number increases with an increase of air velocity, because re-entrainment of a large particle leaves several smaller particles as shown in Fig. 5.

Figure 7 shows the fractional efficiency of re-entrainment, which is interpreted from Fig. 6. To evaluate the experimental results of re-entrainment, the particle diameter, $d_{50}$, at which $50 \%$ of particles in number is re-entrained was defined. Thus-defined diameter is obtained as that corresponding to $50 \%$ fractional efficiency in Fig. 7.

Figure 8 shows the diameter above-defined, $d_{50}$,

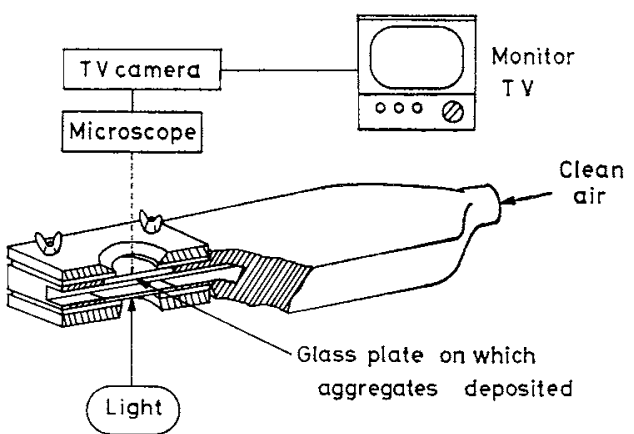

Fig. 4 Experimental apparatus

\section{( Top view)}

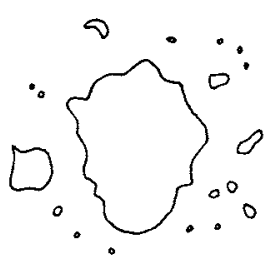

Before

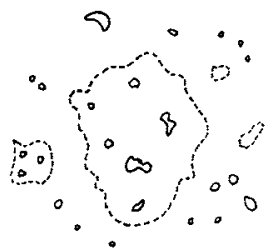

After
Fig. 5 Typical pattern of aggregates observed before and after re-entrainment

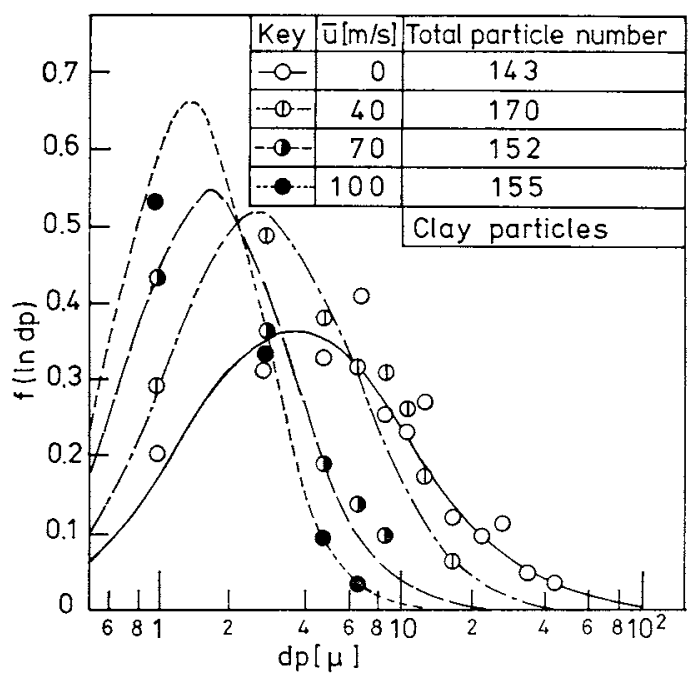

Fig. 6 Change in particle size distribution of aggregate particles due to re-entrainment

against velocity gradient in laminar sub-layer, $\gamma$ together with $\vec{u}$, calculated from Eq. (8). The thickness of laminar sub-layer calculated is also shown in the figure to confirm that the particle is within the layer. The different kinds of lines are calculated ones assuming $y^{*}=0.5 d_{p 1}$ and assuming Rumpf's equation, Eq. (6). In calculating Eq. (6), the porosity of the powder bed packed as loosely as possible was used as $\varepsilon$, the coordination number, $k$, was calculated from the Ridgway-Tarbuck equation ${ }^{5}$, and water volume composing a bridge was calculated from moisture content of a 


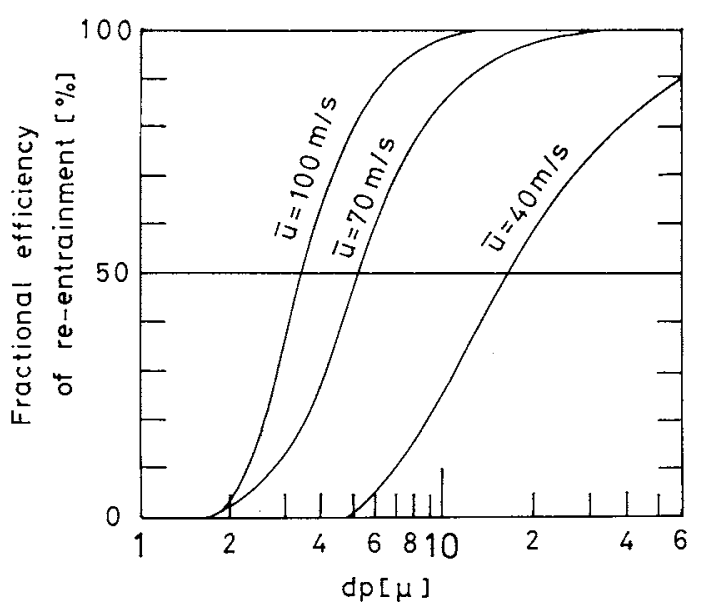

Fig. 7 Fractional efficiency of re-entrainment

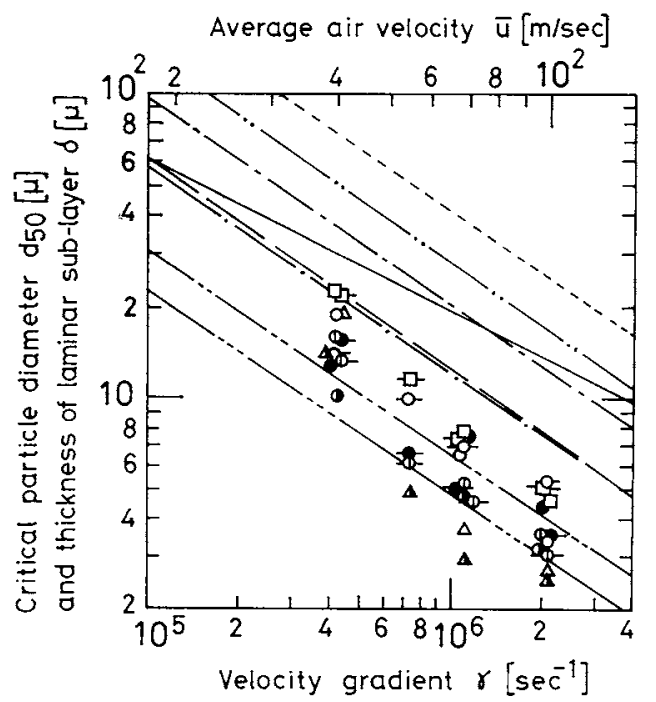

Fig. 8 Relation between $d_{50}$ and $\gamma$, and relation of $u$ and $\delta$

powder bed assuming that all the moisture is equidistributed to form the bridges. These values are shown in Table 1. Because of the uncertainty in evaluating correct adhesive strength, experimental results deviate from the calculated lines. The relation given by Eq. (7), $d_{50} \propto \gamma^{-2 / 3}$, was not made clear by the experiment as shown in Fig. 8 because of both the assumptions made in analysis for simplification and experimental errors.

Apart from the comparison between experiment and calculation, it can be concluded from the experimental results only that large aggregate particles deposited on the media of dust collectors is fairly stable in usual operation where the velocity gradient is not so large as that applied in this study.

To see the effect of the materials of the flat plate, a PVC plate and a glass plate scratched by sandpaper were tested, but almost no difference from the results obtained above was found.

\section{Conclusion}

The mechanism of re-entrainment of small aggregate particles from a flat surface by an air stream was analysed to obtain a macroscopic understanding of the phenomena: firstly, bending stress was found to be important and, secondly, critical diameter to be reentrained was found to be proportional to (velocity gradient) $)^{-2 / 3}$.

The effect of physical properties of primary particles composed of aggregate particles on re-entrainment and the correlation of critical diameter to be re-entrained and (velocity gradient) ${ }^{-2 / 3}$ were not made clear in the experimental work, probably because of the uncertainty in evaluating adhesive force and of the simplification of the analysis.

It was found by experiment that aggregate particles deposited on a flat surface are fairly stable unless the velocity gradient is extremely large.

\section{Appendix}

Derivation of the bending stress and the shearing stress is as follows:

The moment induced by the drag force at the distance $y^{*}(>0)$ as shown in Fig. 2 (a) is given as

$$
M=\int_{y^{*}}^{d_{p}} y d R_{f}
$$

Substitution of Eq. (1) into Eq. (A-1) gives

$$
M=24 \mu \gamma d_{p}{ }^{3} \int_{y^{*} / d_{p}}^{1} \sqrt{(1-Y) Y} Y^{2} d Y
$$

where $Y=y / d_{p}$.

Assuming that the aggregate particle is separated at the circle of diameter $d=2 \sqrt{y^{*}\left(d_{p}-y^{*}\right)}$, the section modulus is given as

$$
\begin{aligned}
Z & =\pi d^{3} / 32 \\
& ={ }_{4}^{\pi}\left[y^{*}\left(d_{p}-y^{*}\right)\right]^{3 / 2}
\end{aligned}
$$

Accordingly, the bending stress, which is the ratio of the moment to the section modulus, can be expressed by Eq. (2). Rearrangement of Eq. (2) by solving the integral part gives the next equation.

$$
\begin{aligned}
\sigma_{b}= & \frac{96 \mu \gamma}{\pi}\left[\frac{\sqrt{\left(1-y^{*} / d_{p}\right) y^{*} / d_{p}}}{192}\left\{15+10\left(\frac{y^{*}}{d_{p}}\right)+8\left(\frac{y^{*}}{d_{p}}\right)^{2}-48\left(\frac{y^{*}}{d_{p}}\right)^{3}\right\}\right. \\
& \left.+\frac{5}{128}\left\{\frac{\pi}{2}+\sin ^{-1}\left(1-2 y^{*} / d_{p}\right)\right\}\right]\left\{\frac{y^{*}}{d_{p}}\left(1-\frac{y^{*}}{d_{p}}\right)\right\}^{-3 / 2}
\end{aligned}
$$

The drag force, which can be obtained by integrating Eq. (A-1), is given as

$$
R_{f}=24 \mu \gamma d_{p}^{2} \int_{y^{*} / d_{p}}^{1} \sqrt{(1-Y) \bar{Y} Y d Y}
$$

where $Y=y / d_{p}$.

If the separation is assumed to take place at the circle of diameter $d=2 \sqrt{y^{*}\left(d_{p}-y^{*}\right)}$, the shearing stress $\tau\left(=R_{f} /(\pi / 4) d^{2}\right)$ can be given by Eq. (4). Equation (4) can be rearranged as follows:

$$
\begin{aligned}
\tau= & \frac{24 \mu \gamma}{\pi}\left[\frac{\sqrt{ }\left(1-y^{*} / d_{p}\right) y^{*} / d_{p}}{24}\left(3-4 \frac{y^{*}}{d_{p}}\right)\left(1+2 \frac{y^{*}}{d_{p}}\right)\right. \\
& \left.+\frac{1}{16}\left\{\frac{\pi}{2}+\sin ^{-1}\left(1-2 \frac{y^{*}}{d_{p}}\right)\right\}\right]\left\{\frac{y^{*}}{d_{p}}\left(1-\frac{y^{*}}{d_{p}}\right)\right\}^{-1}
\end{aligned}
$$




\section{Nomenclature}

$D_{e} \quad=$ equivalent diameter of duct

$d \quad=$ diameter of separation area

$d_{p} \quad=$ diameter of aggregate particle

$d_{p 1} \quad=$ diameter of primary particle

$d_{50} \quad=$ critical particle diameter

$F_{I I}=$ dimensionless adhesive force by water bridge $\left(=H / d_{p 1} \sigma\right)$

$H \quad=$ adhesive force by water bridge

$k=$ coordination number

$M \quad=$ moment

$M_{f} \quad=$ moisture content on dry basis

$R_{f} \quad=$ drag force

$u \quad=$ air velocity

$\bar{u} \quad=$ average air velocity

$Y=$ dimensionless distance $\left(=y / d_{p}\right)$

$y=$ distance above flat surface

$y^{*} \quad=$ distance from flat surface to separated part of aggregate particle as shown in Fig. 2

$=$ section modulus

$=$ velocity gradient

$=$ thickness of laminar sub-layer
$=$ porosity

$=$ viscosity

$=$ kinematic viscosity

$=$ density of fluid

$=$ particle density

$=$ surface tension

$=$ bending stress

$=$ strength of aggregate particle

$=$ shearing stress
$[-]$

[g/cmsec]

$\left[\mathrm{cm}^{2} / \mathrm{sec}\right]$

$\left[\mathrm{g} / \mathrm{cm}^{3}\right]$

$\left[\mathrm{g} / \mathrm{cm}^{3}\right]$

$[$ dyne/cm]

[dyne $\left./ \mathrm{cm}^{2}\right]$

[dyne $\left./ \mathrm{cm}^{2}\right]$

$\left[\right.$ dyne $\left./ \mathrm{cm}^{2}\right]$ [dyne]

$[-]$

[dynecm]

[wt $\%$ ]

[dyne]

$[\mathrm{cm} / \mathrm{sec}]$

$[\mathrm{cm} / \mathrm{sec}]$

$[-]$

[cm]

[cm]

$\left[\mathrm{cm}^{3}\right]$

$[1 / \mathrm{sec}]$

$[\mu],[\mathrm{cm}]$

\title{
Literature Cited
}

1) Fuchs, N. A.: "Mechanics of Aerosols", Pergamon Press, New York (1964).

2) Kanou, T. "Funtairyushi no Kyodou", p. 303, SangyoGijyutsu Center (1977).

3) von Kármán, Th.: Proc. 3rd Intern. Congress Appl. Mech., Stockholm, Part I, 85 (1931).

4) Kousaka, Y., K. Okuyama, A. Shimizu and T. Yoshida: J. Chem. Eng. Japan, 12, 152 (1979).

5) Ridgway, K and K. J. Tarbuck: Brit. Chem. Eng., 12, 384 (1967).

6) Rumpf, H.: Chem. Ing. Tech., 42, 538 (1970).

7) Zimon, A. D.: "Adhesion of Dust and Powder", p. 197, Plenum Press, New York (1969).

\section{POWER CORRELATION FOR ANCHOR AND HELICAL RIBBON IMPELLERS IN HIGHLY VISCOUS LIQUIDS}

\author{
KoJI TAKAHASHI, KUNIO ARAI \\ AND SHOZABURO SAITO \\ Department of Chemical Engineering, Tohoku University, \\ Sendai 980
}

\begin{abstract}
A new power correlation for both anchor and helical ribbon impellers in highly viscous Newtonian liquids is proposed on the basis of a physical model developed from an analytical approximate expression for the drag of a plate in viscous liquids bounded by a plane wall. The correlation, obtained by inserting the empirical factor of geometrical variables in the above expression, shows good agreement with experimental data of power consumption of anchor and helical ribbon agitators obtained in this work and other literature.
\end{abstract}

\section{Introduction}

Liquid agitation is one of the most common unit operations in the chemical industry. For the design of mixing equipment, it is necessary to predict its power consumption. For impellers producing high stress in a small portion of the vessel, such as turbines and propellers, there is fairly extensive information about

Received July 2, 1979. Correspondence concerning this article should be addressed to $S$. Saito. power consumption in the literature ${ }^{9,11}$. However, the power correlations ${ }^{1-4,8)}$ published previously for close-clearance impellers, such as anchor and helical ribbon impellers, are relatively limited. Most of them are empirical and are restricted to particular impellers. In particular, those for helical ribbon impellers are not satisfactory.

In this work, power consumption measurements for anchor and helical ribbon agitators were carried out under laminar flow conditions in Newtonian liquids, 